\title{
Aeolosoma maritimum nov. spec., die erste Salzwasserart aus der Familie Aeolosomatidae (Annelida: Oligochaeta)
}

\author{
W. WESTHEIDE und D. Bunke \\ II. Zoologisches Institut der Universität Göttingen; Göttingen, Deutschland (BRD), \\ und \\ Institut National Scientifique et Technique d'Océanograpbie et de Pêche; \\ Salammbô, Tunesien
}

\begin{abstract}
Aeolosoma maritimum nov. spec, the first sea water species of the family Aeolosomatidae (Annelida: Oligochaeta). Aeolosoma maritimum nov. spec. is the first marine species of the family Aeolosomatidae. The animals were found in the mesopsammal of a Tunisian sandy beach at the Gulf of Tunis; the salinity of the habitat ranged from 29.2 to $34.6 \%$. Investigations on the vertical and horizontal distribution revealed that the species avoids surface layers of the beach. Most individuals occur in the region above subsoil water level, $4 \mathrm{~m}$ off the water line. The most important eidonomic features of $A$. maritimum are: length up to $4 \mathrm{~mm}$; only one single field of cilia on the ventral side of the prostomium; no special ciliary pits; orange-yellow epidermic glands; hair bristles only, 3-5 per bundle, up to $115 \mu \mathrm{m}$; widening of the intestine between the second and third or the third and fourth bundles. No distinct division in zooids could be seen; if paratomy occurs, the new individuals do not separate before the 10th segment. The ecology of the genus Aeolosoma provides an excellent example for the migration of limnic elements into marine habitats.
\end{abstract}

\section{EINLEITUNG}

Bei Untersuchungen an der interstitiellen Fauna tunesischer Sandstrände des Mittelmeeres wurden im Sommer 1969 mehrere Individuen einer Aeolosoma-Art entdeckt. Die Tiere konnten im gleichen Biotop auch im Februar und März 1970 wiedergefunden werden. Habituell lassen sie sich in keine der bisher bekannten und durch BUNKE (1967) eingehend behandelten Species der Gattung einfügen: sie repräsentieren eine neue Art.

Besonderes Interesse verdient Aeolosoma maritimum nov. spec. in ökologischer Hinsicht. Die Familie Acolosomatidae mit den Gattungen Aeolosoma Errenberg, Rbeomorpha RutTNER-Kolisko und Hystricosoma MichaELSEN enthält nur typische Süßwasseroligochaeten, von denen drei Arten gelegentlich in mesohalinen Brackwasserbereichen angetroffen werden. Aeolosoma maritimum ist die erste Species, die einen vollmarinen Biotop bewohnt. 


\section{MATERIAL UND METHODE}

Die Tiere wurden mit einer nach der UHLIG-Methode arbeitenden Apparatur (Westheide \& Sсhмidt 1969) aus dem Sand extrahiert. Die Sandproben sind nach der allgemein im II. Zoologischen Institut in Göttingen angewandten Methodik (Schmid 1968) aus dem Strand entnommen worden. Für die Korngrößenanalysen wurden Siebgrößen nach den Empfehlungen des "Mantual for the Study of Meiofauna" (Hulings 1971) verwendet. Insgesamt konnten 22 Exemplare gefunden werden, von denen 12 - ausschließlich im lebenden Zustand - untersucht wurden.

\section{FUNDORT UND OKOLOGISCHE DATEN}

A. maritimum wurde an der Mittelmeerküste, Tunesien, Golf von Tunis, Sandstrand bei Amilcar (etwa 25 m nordwestlich vom Restaurant „Amphitrite") gefunden. Locus typicus der neuen Art ist das Mesopsammal eines Sandstrandes mit schwachen Gezeiten (durchschnittlich 0,2 m). Die Tiere bewohnen in sehr geringer Abundanz eine etwa $4 \mathrm{~m}$ breite Region des Strandes zwischen Brandungszone und oberem Sandhang. Vertikale und horizontale Verteilung gehen aus den Abbildungen $1 \mathrm{~A}$ und $\mathrm{B}$ hervor. A. maritimum meidet die oberen Sandschichten. Die tiefste Fundstelle lag $0,8 \mathrm{~m}$ unter der Oberfläche des Strandes. Bevorzugter Lebensraum sind im Winter die stark feuchten oder schon wassergesättigten Zonen oberhalb des Grundwassers. 5 Individuen wurden im Grundwasserbereich gefangen. Fundstellen aus dem Sommer liegen auch in einer Zone geringerer Feuchtigkeit.

Das Substrat ist ein grober, aus verschiedenen Mineralien bestehender Sand mit nur sehr wenigen organischen Hartteilen und relativ geringen Mengen Detritus. Die Korngrößen-MD-Werte von 2 Fundstellen liegen zwischen 235 und $375 \mu \mathrm{m}$, die Sortierungskoeffizienten haben die Werte 1,23 und 2,34. Salzgehaltsmessungen konnten nur im Grundwasserbereich der Fundstellen gemacht werden: 34,6; 29,4; 29,2\% (Juli 1969); 34,6; 34,4; 34,1;31,1\% (März 1970). Die Begleitfauna der Feuchtsandzone ist arten- und individuenreich. Charakteristische Formen sind die beiden typischen Sandlückenpolychaeten Hesionides gohari HARTMANN-SCHRÖDER und Hesionides arenaria FRIEDRICH, der Archianelide Diurodrilus benazzii GERLACH, der Oligochaet Marionina preclitellochaeta Nrelsen \& Christensen und eine Derocheilocaris-Art aus der Crustaceen-Gruppe der Mystacocarida.

\section{ORGANISATION}

Für die Familie Aeolosomatidae ist ungeschlechtliche Vermehrung durch Paratomie eine charakteristische Erscheinung. An den 12 genauer untersuchten Exemplaren der neuen Art konnte jedoch bisher eine Gliederung in eine Aufeinanderfolge von Zooiden nicht exakt festgestellt werden. Allerdings läßt die Ausdehnung des Mitteldarmbereiches mit einiger Sicherheit annehmen, daß die 1. Zooide wenigstens 10 oder 

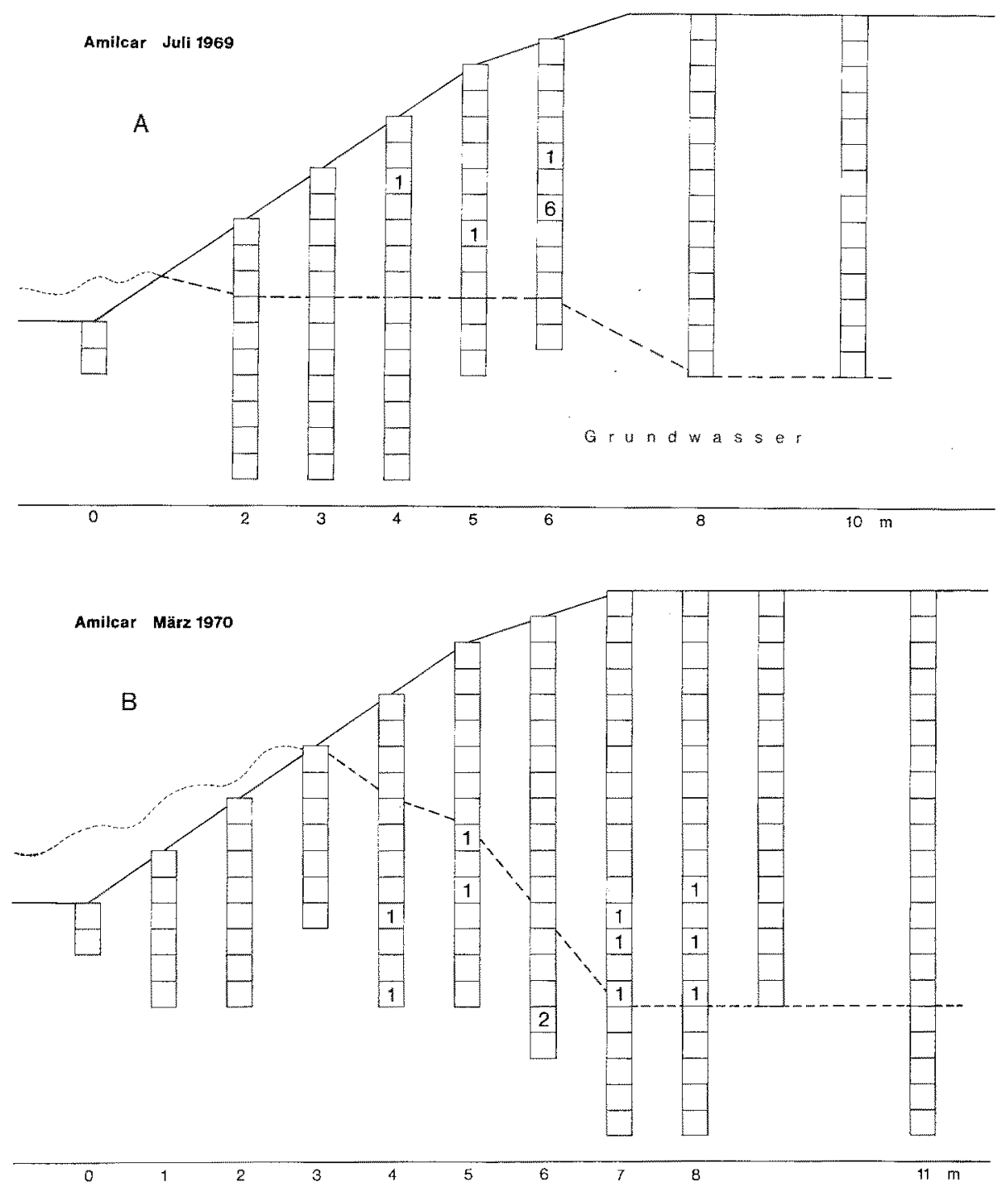

Abb. 1: Aeolosoma maritimum nov. spec. Verteilungsmuster im Strand von Amilcar (locus typicus). Strandprofil stark überhöht gezeichnet; auf der linken Seite der Strandknick und Ubergang in das Sublitoral, auf der rechten Seite Sandhang; unterhalb der gestrichelten Linie wassergesättigter Grundwasserbereich. Jedes Kästchen entspricht einem Sandzylinder von $5 \mathrm{~cm} H$ the und $50 \mathrm{~cm}^{3}$ Inhalt. $A$ Horizontales und vertikales Verteilungsbild yom Juli 1969. $B$ Verteilungsbild vom März 1970

11 Borstenbündelpaare aufweisen müssen. Es ist jedoch auch denkbar, daß bei dieser Art eine andere Form der ungeschlechtlichen Vermehrung vorliegt.

Die Gesamtzahl der Borstensegmente betrug bei den einzelnen Exemplaren: 19, 26 (Juli 1969); 8, 12, 13, 13, 13, 14, 14, 15, 18 und 21 (Februar, März 1970). 
Die Länge eines Tieres mit 13 Borstensegmenten beträgt in gestrecktem Zustand 2,2 mm; ein Exemplar mit 26 Metameren ist etwa $4 \mathrm{~mm}$ lang. Die Breite schwankt je nach Kontraktionszustand zwischen 40 und $50 \mu \mathrm{m}$.

Die Farbdrüsen des Integuments sind unregelmäßig gelappte und ausgebuchtete, rundliche oder ovale Gebilde. Die Farbe ist ein helles Orangegelb oder Goldgelb. Die Größe ist unterschiedlich: 9-14 $\mu \mathrm{m}$. Auf der Dorsalseite des Vorderendes finden sich zumeist kleinere Hautdrüsen als auf den Segmenten. Einzelne Drüsen besitzen kleine

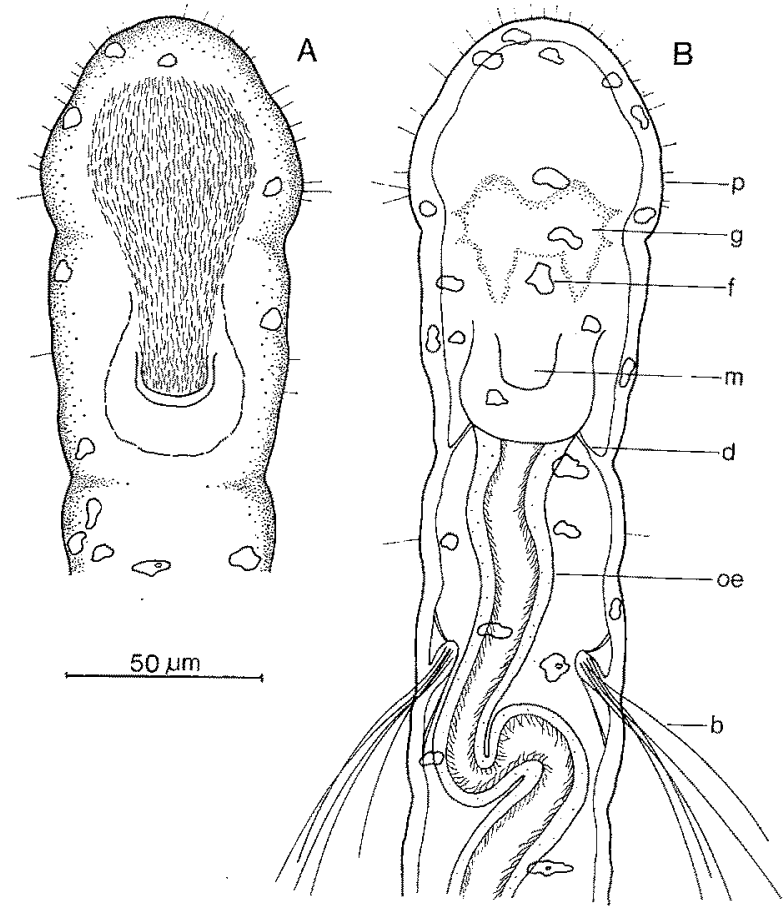

Abb. 2: Aeolosoma maritimum nov. spec. Vorderende. A Aufsicht auf die Ventralseite des Prostomiums; Ausdehnung des Wimperfeldes. $B$ Vorderende in Auf- und Durchsicht; $b 1$. ventrales Borstenbündel, $d$ Dissepiment, $f$ Farbdrüse des Integuments, $g$ Gehirn, $m$ Mundöffnung, oe Oesophagus, $p$ Prostomium

Einschlüsse. Die Zahl ist sehr variabel; zumeist zählt man zwischen zwei Borstenbündelpaaren ungefähr 20 Farbdrüsen. Ein Individuum mit 15 Borstensegmenten hatte jedoch nur etwa 10 über den gesamten Körper verteilte Hauteinschlïsse, während zwei Exemplare - mit 8 bzw. 21 Borstensegmenten - völlig farblos waren und keine Hautdrüsen besaßen. Das Fehlen der Farbdrüsen im Integument einzelner Individuen normalerweise gefärbter Arten ist jedoch häufiger in der Familie Aeolosomatidae beobachtet worden (BUNKE 1967) und kann daher nicht als artspezifische Besonderheit betrachtet werden.

Der Kopflappen hebt sich in schwacher Rundung vom Körperstamm ab; seine breiteste Stelle beträgt etwa $65 \mu \mathrm{m}$. An der Frontalseite des Kopfes stehen zahlreiche, 
sehr kleine Tasthärchen. Einzelne, etwas größere Tasthaare finden sich auch auf den Segmenten. Das Wimperfeld ist auf die Ventralseite des Kopfes beschränkt. Es paßt sich ungefähr der Form des Prostomiums an; vorn hat es seine größte Breite und verschmälert sich nach hinten, um in den Mundyorraum hineinzulaufen. Das Prostomium weist auf der Höhe des Gehirns jederseits eine Vertiefung auf, doch konnten echte Wimpergruben, wie sie für die Mehrzahl der Aeolosoma-Arten charakteristisch sind,

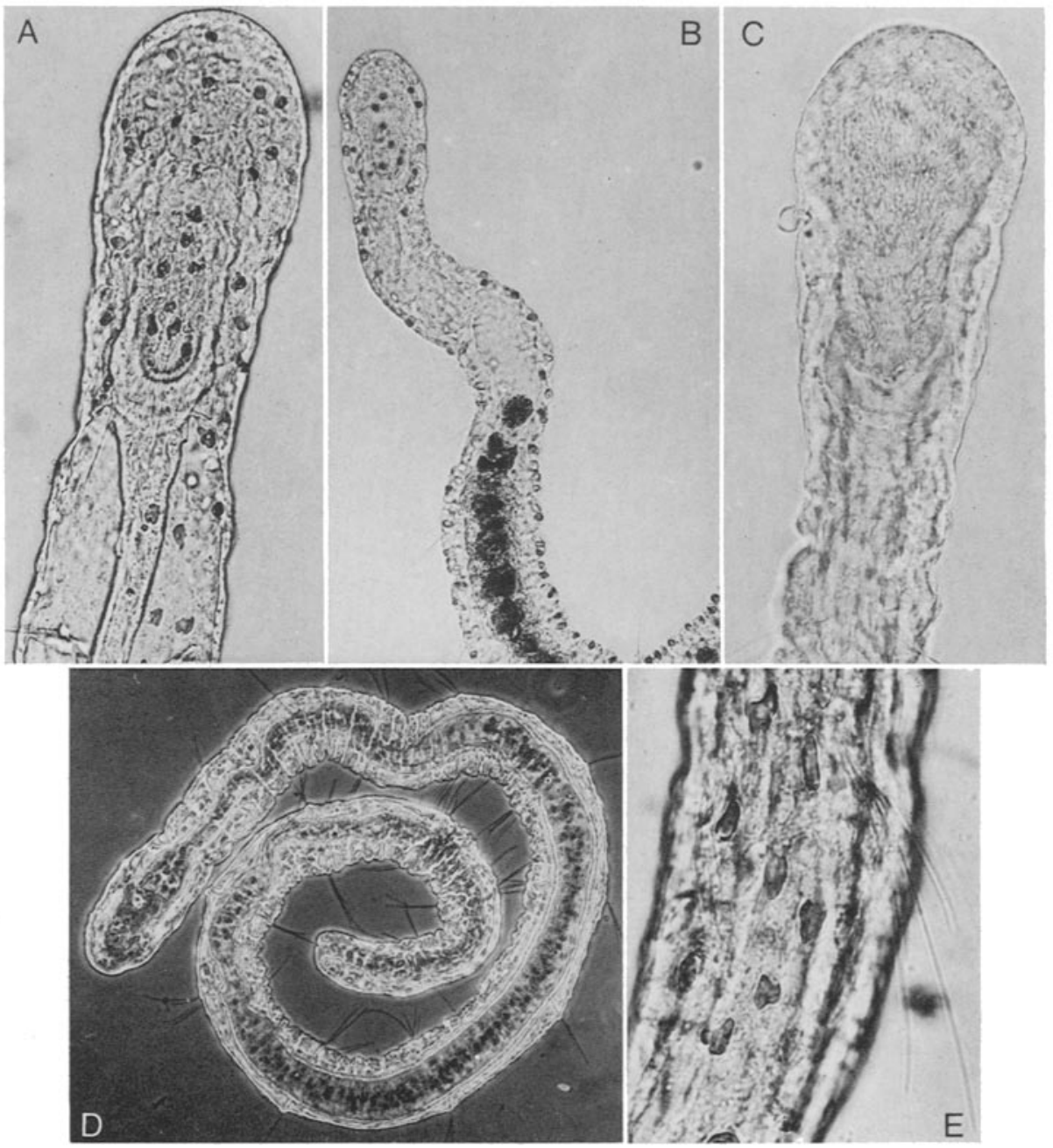

Abb. 3: Aeolosoma maritimum nov. spec. Mikrophotos. A Prostomium, Mund- und vordere Oesophagusregion in leicht gestrecktem Zustand. Gehirn, Mundöffnung, Dissepiment und Hautdrüsen sichtbar. $B$ Vordere Hälfte eines Tieres. Habitus mit natürlichen Dimensionen. $C$ Vorderende eines Individuums ohne Farbdrïsen von ventral mit Wimperfeld. $D$ Vollständiges Tier; Demonstration der Borsten und des Darmkanals im Phasenkontrast. $E$ Ausschnitt aus der mittleren Körperregion mit Borstenbündel und Farbdrüsen 
nicht gesehen werden. An den Kopflappen schließt sich die Mundregion mit einer etwa $20 \mu \mathrm{m}$ breiten Mundöffnung an. Diese ist caudal durch einen breiten Wulst begrenzt. Der Abstand zum Kopfrand beträgt $90 \mu \mathrm{m}$.

Die homonomen Borstensegmente haben eine Länge von ungefähr $90 \mu \mathrm{m}$. Sie sind durch Einschnürungen in 3 etwa gleichgroße Abschnitte gegliedert. Zwischen zweitem und letztem Drittel stehen ein ventrales und ein dorsales Borstenbündelpaar. Die Borstenbewaffnung ist recht gleichmäßig: 3, 4 oder zumeist 5 feine Haarborsten bilden ein Bündel. Kurze, charakteristisch geformte, sogenannte Sigmoidborsten, die eine größere Anzahl anderer Aeolosoma-Arten auszeichnen, fehlen bei der vorliegenden Art sowohl in den Dorsal- als auch in den Ventralbündeln. Die Haarborsten sind in ihrem basalen Teil leicht S-förmig gebogen und am distalen Ende auf der Konvexseite über eine längere Strecke fein gezähnt (Phasenkontrast-Olimmersion). Die Borsten eines Bündels sind nicht alle gleich lang; maximal erreichen sie 100-115 $\mu \mathrm{m}$.

Der vordere schmale Darmabschnitt verbreitert sich zwischen dem zweiten und dritten oder dritten und vierten Borstenbündelpaaren zum Mitteldarm. Nur hinter dem Mundabschnitt ist ein vollständiges Dissepiment vorhanden. Die bei Lebendbeobachtung in der Regel deutlich sichtbaren Nephridien der Aeolosoma-Arten wurden bei der vorliegenden Form nicht erkannt. Sie fehlen entweder generell oder sind schwächer ausgebildet, eine Tendenz, die auch bei zahlreichen anderen Arten dieser Gattung nachzuweisen ist. Das Gehirn ist gut sichtbar und hat die von anderen Aeolosoma-Species bekannte breite bilobe Form. Allen Individuen von A. maritimum fehlten Geschlechtsorgane.

\section{DISKUSSION}

Auf Grund der Farbe der Hautdrüsen ließe sich Aeolosoma maritimum nov. spec. sowohl in die 1. als auch 2. Artengruppe dieser Gattung (Bunke 1967) einordnen, die orange- bis braunrote bzw. goldgelbe bis blaugrüne Drüsen besitzen.

Die relativ langen Borsten im Vergleich zum Körperdurchmesser sowie die unregelmäßig lappige Gestalt der Hautdrüsen legen zunächst eine Zugehörigkeit zu den Formen der 2. Artengruppe nahe. Von den zehn Arten dieser Reihe kommen jedoch für eine Abgrenzung zur vorliegenden Art lediglich Aeolosoma aureum Marcus und Aeolosoma flavum STolc in Betracht, die im Typus der Borsten mit dieser ubereinstimmen und in den Körperdimensionen und der Hautdrüsenfarbe vergleichbar sind. $A$. aureum besitzt jedoch goldgrünes, glänzendes Hautsekret und ist ferner durch deutliche Wimpergruben am Prostomium sowie bis zu $150 \mu \mathrm{m}$ lange Borsten klar von der dargestellten Art unterscheidbar. In der Farbe der Hautdrüsen stimmt A. maritimum nov. spec. am besten mit $A$. flavum überein, die gelbes Sekret aufweist. Diese Art wurde auch einmal im tieferen Mesopsammal, allerdings im Süßwasserbereich, gefunden (Bunke 1967). Neben anderen Abweichungen, die die Länge der Borsten und die Körperbreite betreffen, liegt die wichtigste Differenz beider Arten in der Segmentzahl der 1. Zooide, die bei A. flavum 7 Borstenbündelpaare, bei der vorliegenden Form jedoch sicher 10 oder mehr besitzen. 
Das häufig orange-gelbe Hautsekret von A. maritimum nov. spec. macht darüber hinaus noch eine Abhebung von ähnlichen Arten aus der roten Formenreihe (1. Artengruppe) notwendig. Hier stimmen $A$. litorale Bunke und A. kasbyapi Stephenson auffällig durch das Fehlen eindeutiger Wimpergruben am Prostomium mit unserer Art überein. Ferner haben sie nur einen einzigen Borstentyp (Haarborsten). Dennoch ergibt sich auch hier eine klare Abgrenzung durch die rote Farbe der Hautdrüsen dieser Arten. Sie wird noch unterstrichen durch die geringere Körperlänge und Segmentzahl der 1 . Zooide bei $A$. kashyapi sowie die beträchtlich größere Körperbreite und größere Anzahl und Länge der Borsten bei $A$. litorale.

Außer diesen habituellen Unterschieden zu den erwähnten Arten ist A. maritimum durch eine bisher einzig dastehende ökologische Besonderheit auch von allen übrigen bekannten Aeolosoma-Arten eindeutig abgetrennt: Die neue Species besiedelt einen marinen Bereich.

Aus den Angaben von BunKe geht hervor, daß die meisten Formen der Gattung (etwa 14 Arten) typische Bewohner des limnischen Phytals sind. 5 Arten gehören sowohl dem Phytal wie auch dem limnischen Mesopsammal an. Nur zwei Species wurden ausschließlich im interstitiellen Sandgefüge gefunden. Bei zwei Arten besteht eine Tendenz zur Besiedlung auch von Brackwasserbiotopen. So wird A. variegatum VEJDovsKy von LAAKso (1969) für die Südwestküste Finnlands (Lappohja) (Salzgehalt bis $6 \%$ ) angegeben. Bunke (1967) hat A. litorale Bunke in der Schlei bei Schleswig, an der Kieler Förde und in Salzwiesenufern von Südwestfinnland in brackigen (bis $5 \%$ Salzgehalt) mesopsammalen Lebensräumen nachgewiesen. Zwischen $A$. litorale und $A$. maritimum bestehen daher nicht nur morphologische Ubereinstimmungen, beide Arten weisen auch engere ökologische Beziehungen zueinander auf.

In historisch-zoogeographischer Hinsicht ist A. maritimum ein Einwanderer aus dem limnischen Bereich. Während der Vorstoß mariner Elemente der Mikrofauna in das limnische Mesopsammal durch zahlreiche Beispiele zu belegen ist (Ax 1956, RIEmann 1968), wird von Remane (1950) der Prozentsatz limnischer Elemente im marinen Sandlückensystem der Kieler Bucht mit nur etwa $10 \%$ angegeben. An anderen Küsten mit höherem Salzgehalt und einem artenreicheren Mesopsammon dürfte die Prozentzahl noch geringer sein. Unter diesen wenigen limnischen Einwanderern stellen die Oligochaeten wohl die stärkste Gruppe.

Die ökologischen Daten der Gattung Aeolosoma erlauben es nun, eine Einwanderungslinie limnischer Oligochaeten in das marine Mesopsammal aufzuzeigen. Am Anfang dieses Weges stehen Arten, die teilweise oder zeitweilig in das limnische Sandlückensystem eindringen, z. B. A. bemprichi, A. travancorense und A. byalinum. Die zweite Station auf dem Einwanderungsweg wird durch Arten repräsentiert, die ausschließlich Bewohner dieses Lebensraumes sind, wie A. psammophilum und A. litorale. Von hier aus erfolgt ein Vordringen in brackige lenitische Uferregionen durch $A$. litorale. Aeolosoma maritimum hat schließlich einen euryhalinen Biotop in den geschützten Regionen eines marinen Sandstrandes erreicht. 


\section{ARTDIAGNOSE}

Aeolosoma maritimum nov. spec. Bis $4 \mathrm{~mm}$ langer Körper; Breite $40-50 \mu \mathrm{m}$. Epidermisdrüsen gelb bis orange gefärbt. Einheitliches Wimperfeld nur auf der Ventralseite des Prostomiums, keine besonderen Ciliengruben. Mitteldarmerweiterung zwischen den 2. und 3. oder 3. und 4. Borstenbündeln. Der Vorgang der ungeschlechtlichen Vermehrung ist ungeklärt; die 1. Zooide haben jedoch wenigstens 10 Borstensegmente, falls Paratomie vorliegt. Nur sehr feine, bis zu $115 \mu \mathrm{m}$ lange Haarborsten, 3-5 in einem Bündel.

Exemplare aus der Typenreihe befinden sich im Zoologischen Museum der Universität Hamburg.

\section{ZUSAMMENFASSUNG}

1. Aeolosoma maritimum nov. spec. wurde im Mesopsammal eines Sandstrandes am Golf von Tunis gefunden; der Salzgehalt im Porenwasser der Probestellen lag zwischen 29,2 und $34,6 \%$.

2. Die Tiere meiden die oberflächlichen Regionen des Strandes; die meisten Individuen wurden unmittelbar über oder im Grundwasserbereich gefangen, in einer Entfernung von etwa $4 \mathrm{~m}$ von der Wasserlinie.

3. Die Art repräsentiert somit die erste marine Form aus der Oligochaeten-Familie Aeolosomatidae. Aus den ökologischen Daten anderer Aeolosoma-Species kann ein Einwanderungsweg aus dem limnischen Mesopsammal über das Brackwasser in das marine Sandlückensystem rekonstruiert werden.

4. Merkmale der äußeren und inneren Organisation weisen $A$. maritimum ebenfalls als eine gut definierte Art aus.

Danksagungen. Der Aufenthalt in Tunesien wurde einem von uns (W. WESTHEIDE) durch finanzielle Unterstïtzung des Mediterranean Marine Sorting Center in Salammbô und Khereddine (Direktoren: Dr. N. C. Hulrngs und Dr. R. P. HrgGINs) und der Fritz-ThyssenStiftung, Köln, ermöglicht. Unterbringung, Arbeitsplatz und technische Hilfe wurden durch Mme Dr. F. KTARI, Direktorin des Institut National Scientifique et Technique d'Océanographie et de Pêche in Salammbô gewährt. Vielfältige freundschaftliche Unterstützung leistete Dr. Hrgarns. Ihnen allen sei herzlich gedankt.

\section{ZITIERTE LITERATUR}

Ax, P., 1957. Die Einwanderung mariner Elemente der Mikrofauna in das limnische Mesopsammal der Elbe. Zool. Anz. (Suppl. Bd) 20, 428-435.

Bunke, D., 1967. Zur Morphologie und Systematik der Aeolosomatidae BedDard 1895 und Potamodrilidae nov. fam. (Oligochaeta). Zool. Jb. (Abt. Syst. Okol. Tiere) 94, 187-368.

Hulings, N. C. (Hrsg.), 1971. A manual for the study of meiofauna. (Im Druck.)

LaAkso, M., 1969. New records of aquatic Oligochaeta from Finland. Annls Zool. fenn. 6, 348-352.

Remane, A., 1950. Das Vordringen limnischer Tierarten in das Meeresgebiet der Nord- und Ostsee. Kieler Meeresforsch. 7, 5-23. 
Riemann, F., 1968. Die Mikrofauna subterraner Lebensräume in Grenzbereich Meer-Süßwasser. Naturw. Rdsch., Stuttg. 21, 198-203.

Scemidt, P., 1968. Die quantitative Verteilung und Populationsdynamik des Mesopsammons am Gezeiten-Sandstrand der Nordseeinsel Sylt. I. Faktorengefüge und biologische Gliederung des Lebensraumes. Int. Revue ges. Hydrobiol. 53, 723-779.

Westheide, W. \& Schmidt, P., 1969. Von der Kleintierwelt im Meeresstrand. I. Fang und Untersuchung. Mikrokosmos 58, 257-262.

Anschrift des erstgenannten Autors: Dr. W. Westherde

II. Zoologisches Institut

Universität Göttingen

34 Göttingen

Berliner Straße 28

Deutschland (BRD) 